\title{
Influence of the Neutron Star 1E 161348-5055 in RCW 103 on the Surrounding Medium
}

\author{
E. M. Reynoso, ${ }^{1,2,4}$ A. J. Green, ${ }^{1}$ S. Johnston, ${ }^{1}$ W. M. Goss, ${ }^{3}$ G. M. Dubner, ${ }^{2, *}$ \\ and E. B. Giacani ${ }^{2, *}$ \\ ${ }^{1}$ School of Physics, University of Sydney, Sydney NSW 2006, Australia \\ ${ }^{2}$ Instituto de Astronomía y Física del Espacio, CC 67, Suc 28, 1428 Buenos Aires, Argentina \\ ${ }^{3}$ National Radio Astronomy Observatory, P. O. Box 0, Socorro, NM 87801, USA \\ ${ }^{4}$ Author to whom correspondence should be addressed (e-mail: ereynoso@iafe.uba.ar)
}

Received 2003 September 3, accepted 2003 September 23

\begin{abstract}
We have carried out a study of the neutral hydrogen in the direction of the X-ray source 1E 1613485055 , a compact central object (CCO) located in the interior of the supernova remnant (SNR) RCW 103. The HI $21 \mathrm{~cm}$ line observations were carried out using the Australia Telescope Compact Array, complemented with single dish data from the Parkes radio telescope to recover information at all spatial scales. We derive a distance to RCW 103 of $3.1 \mathrm{kpc}$, in agreement with previous distance measurements. We have also detected a small hole in the Hi emission which is positionally and kinematically coincident with the location of the CCO which confirms the association between the SNR and the CCO. This is the third case of a depression in HI emission seemingly associated with CCOs in SNRs. The characteristic parameters of the holes such as their size, eccentricity and evacuated mass are similar in all three cases. We estimate the absorbing Hi column density towards $1 \mathrm{E} 161348-5055$ to be $\sim 6 \times 10^{21} \mathrm{~cm}^{-2}$, a value compatible with a blackbody solution for the CCO X-ray emission. However, the implied temperature and luminosity are very high compared to most neutron stars. Moreover, the strong long-term variability in X-rays favours the hypothesis that 1E 161348-5055 is an accreting binary source rather than an isolated, cooling neutron star. An analysis of the continuum image obtained at $1.4 \mathrm{GHz}$ from these observations shows no trace of a pulsar wind nebula around 1E 161348-5055, in spite of it being a young object.
\end{abstract}

Keywords: stars: neutron — supernova remnants — ISM: individual: RCW 103 - X-rays: individual: 1E 161348-5055 — spectral lines: neutral hydrogen.

\section{Introduction}

X-ray observations from the past few years have revealed the existence of a large variety of Galactic point-like sources, many of them identified with neutron stars but presenting very different observational properties. These unresolved sources, which appear either isolated or in the interior of supernova remnants, have no radio counterpart and very high X-ray to optical flux ratios. About half of these sources show X-ray pulsations with periods between 6 and 12 seconds (anomalous X-ray pulsars, AXPs) and can even present sporadic strong $\gamma$-ray emission (soft gamma-ray repeaters, SGRs). The rest of these sources are generally called either isolated neutron stars (INSs) if they are not associated with any other object in the sky, or central compact objects if they appear projected on the interior of a SNR (see a recent review by Pavlov et al. 2002).

The nature of most CCOs is still unclear. Brazier \& Johnston (1999) explain them as normal pulsars with unfavourable radio beaming, while the soft thermal X-rays

\footnotetext{
*Member of the Carrera del Investigador Científico, CONICET, Argentina.
}

would be easily detected due to their almost isotropic emission. On the other hand, Vasisht et al. (1997) and Frail (1998) conclude that CCOs are neutron stars (NSs) born with long initial periods and high magnetic fields $\left(B>10^{14} \mathrm{G}\right)$, and thus would be related to AXPs and SGRs. However, Geppert, Page, \& Zannias (1999) suggest that CCOs are fast-spinning, weakly-magnetised sources. The X-ray emission from CCOs is generally explained as thermal radiation from cooling NSs (e.g Zavlin, Trümper, \& Pavlov 1999), with typical temperatures of a few times $10^{6} \mathrm{~K}$, as inferred from their thermal-like spectra.

In a recent $\mathrm{HI}$ study towards the bilateral SNR G296.5+10.0, Giacani et al. (2000) found that the associated CCO, 1E 1207.4-5209, lies near the centre of a small HI depression located at the same systemic velocity as the SNR. The authors propose that the depression is due to self-absorption of a cool Hi cloud just to the foreground of a hotter volume of gas surrounding the $\mathrm{CCO}$, and heated by its X-ray flux. We have begun a systematic search for similar traces in the neutral gas around other CCOs. The observations towards the X-ray point source RX J08224300 in Puppis A revealed an Hi structure consisting of a nearly circular minimum coincident with the $\mathrm{CCO}$ plus 
two aligned lobe-like depressions that appear to emerge from the CCO (Reynoso et al. 2003). The two lobes appear to have been formed by a combination of the proper motion of the $\mathrm{CCO}$ and the ejection of a collimated outflow. In this paper, we present the results obtained for $1 \mathrm{E}$ 161348-5055, the CCO associated with the SNR RCW 103 (G332.4-0.4).

At radio wavelengths, RCW 103 appears as an almost complete, circular, $8^{\prime}$ diameter shell (Caswell et al. 1980). High-resolution radio polarimetric data reveal an approximately east-west alignment of the magnetic field which could be helping to limit the expansion north-south (Dickel et al. 1996). The brightening of the rim in the northern and southern sides, that hints for an incipient bilateral barrel shape for this SNR, could be related to this frozen-in magnetic field. Based on Hi absorption measurements at $21 \mathrm{~cm}$, Caswell et al. (1975) suggest a distance of $3.3 \mathrm{kpc}$. Optical filaments are seen toward the brighter regions of the radio shell (van den Bergh, Marscher, \& Terzian 1973; Ruiz 1983), and observations at infrared wavelengths show evidence for interaction with a dense interstellar medium (ISM), particularly to the south (Oliva, Moorwood, \& Danziger 1990; Burton \& Spyromilio 1993; Oliva et al. 1999). An optical expansion study of RCW 103 (Carter, Dickel, \& Bomans 1997) indicates that this SNR is probably about 2000 years old, based on an assumed distance of $3.3 \mathrm{kpc}$. However, optical extinction studies suggest distances around $6.5 \mathrm{kpc}$ (Leibowitz \& Danziger 1983; Ruiz 1983).

Soft X-ray emission from RCW 103 was first detected with the Einstein Observatory (Tuohy et al. 1979), indicating a close correlation with the non-thermal radio emission. Also, a faint, point X-ray source, 1E 1613485055 , was located near the centre of the SNR (Tuohy \& Garmire 1980). The lack of optical or radio counterparts led Tuohy et al. (1983) to propose that this point source was a thermally radiating CCO. However, subsequent observations with the Einstein IPC and ROSAT failed to confirm the existence of this source (Becker et al. 1993). Finally, Gotthelf, Petre, \& Hwang (1997) detected hard X-rays from the elusive CCO using ASCA. They found that the spectral characteristics were incompatible with a simple cooling NS model. Follow-up observations (Gotthelf, Petre, \& Vasisht 1999) confirmed that 1E 161348-5055 manifests long-term variability, explaining the non-detections after its discovery. There are strong indications that $1 \mathrm{E} 161348-5055$ is part of a binary system (see section 4.1).

In this paper, we present radio contiuum at $1.38 \mathrm{GHz}$ and $\mathrm{HI}_{\mathrm{I}} \lambda 21 \mathrm{~cm}$ observations carried out with the Australia Telescope Compact Array (ATCA) towards the SNR RCW 103.

\section{Observations and Data Reduction}

Interferometric observations were obtained with the ATCA during one session of $12 \mathrm{~h}$ with the $750 \mathrm{~A}$ array (baselines from 76.5 to $735 \mathrm{~m}$ ), on 2002 January 22, and one session of $12 \mathrm{~h}$ with the EW 367 array (baselines from 46 to $367 \mathrm{~m}$ ) on 2002 April 1 . The antennas were pointed at $\mathrm{RA}=16^{\mathrm{h}} 17^{\mathrm{m}} 30^{\mathrm{s}} \cdot 0$, Dec $=-51^{\circ} 0^{\prime} 0^{\prime \prime}(\mathrm{J} 2000)$. A correlator configuration of 1024 channels, covering a total bandwith of $4 \mathrm{MHz}$ centred at $1420 \mathrm{MHz}$, was used. The corresponding velocity resolution at this frequency is $1 \mathrm{~km} \mathrm{~s}^{-1}$. Continuum data were obtained simultaneously with a bandwidth of $128 \mathrm{MHz}$ centred at $1384 \mathrm{MHz}$. The source PKS B1934-638 was used for primary flux density calibration, while phases were calibrated with PKS B1657-56.

The data were processed with the MIRIAD software package (Sault, Teuben, \& Wright 1995). To subtract the continuum component from the Hi data set, a linear baseline was fitted to 370 line-free channels. The final Hi cube was constructed with a $15^{\prime \prime}$ cell size, covering an area of $64^{\prime} \times 64^{\prime}$ and keeping 350 channels from -150 to $+138 \mathrm{~km} \mathrm{~s}^{-1}$. Sidelobes were supressed over an area of $34^{\prime} \times 34^{\prime}$. The image was cleaned and convolved with a $50^{\prime \prime}$ Gaussian beam. The continuum image was constructed with the same geometry and angular resolution as the Hi data. After cleaning and restoring with a 50" Gaussian beam, a sensitivity of $\sigma \simeq 5.5 \mathrm{mJy}^{\text {beam }}{ }^{-1}$ was achieved.

To recover structures at shorter spatial frequencies, the ATCA HI data were combined in the $u v$ plane with single dish data from the Southern Galactic Plane Survey (SGPS, McClure-Griffiths et al. 2001) obtained with the Parkes telescope. In order to match the intensity units in both sets, a factor of 257.5 was applied to the ATCA data cube to convert flux densities to brightness temperatures. No tapering was applied to the low resolution cube. The rms of the combined image is $2 \mathrm{~K}$ per channel.

\section{Results}

\subsection{Absorption Study}

To constrain the systemic velocity of RCW 103, we analysed the $\mathrm{HI}$ absorption against the strong radio continuum emission from the remnant. The radio contimuum image obtained with ATCA at the frequency of $1384 \mathrm{MHz}$ in a field of about 45' around RCW 103 is shown in Figure 1. According to the equation of radiative transfer, the emission $T_{\mathrm{b}_{v}}$ at a velocity $v$ measured by an interferometer is given by

$$
T_{\mathrm{b}_{v}}=T_{\mathrm{s}}\left(1-e^{-\tau_{v}}\right)+T_{\mathrm{c}} e^{-\tau_{v}},
$$

where $T_{\mathrm{s}}$ and $\tau_{v}$ are the spin temperature and the optical depth of the intervening HI gas, and $T_{\mathrm{c}}$ is the background continuum emission. Since we have subtracted the radio continuum from our Hi data, then Equation (1) can be reduced to

$$
T_{\mathrm{L}_{v}}=\left(T_{\mathrm{s}}-T_{\mathrm{c}}\right)\left(1-e^{-\tau_{v}}\right),
$$

where $T_{\mathrm{L}_{v}}=T_{\mathrm{b}_{v}}-T_{\mathrm{c}}$. Equation (1) can be simplified to a single unknown if $T_{\mathrm{c}} \gg T_{\mathrm{s}}$. This condition can be accomplished by using only interferometric data, since in such way the extended $\mathrm{HI}$ emission is filtered out. 


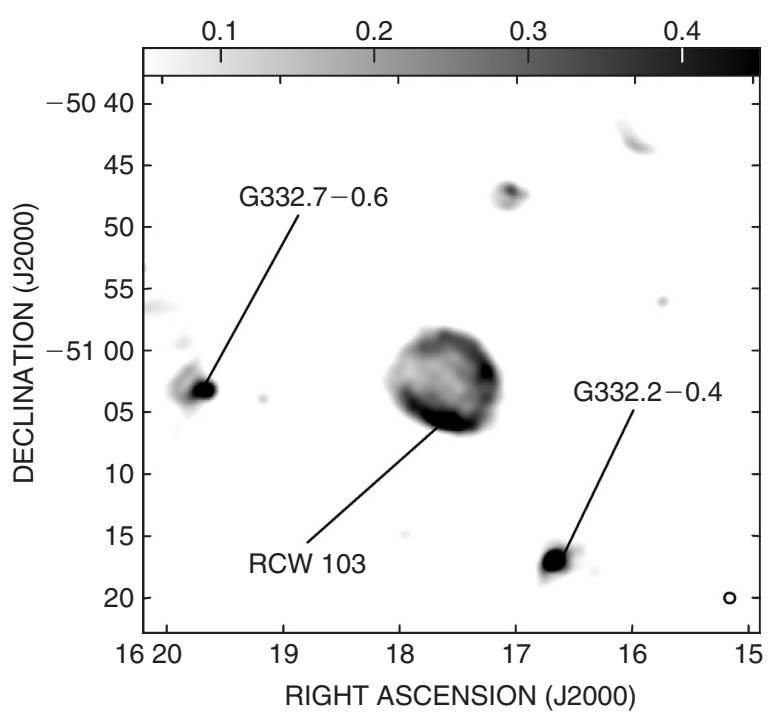

Figure 1 Radio continuum image centred at RCW 103, obtained with the ATCA at $1384 \mathrm{MHz}$. The gray scale is shown on top of the image in units of Jy beam ${ }^{-1}$. The beam, $50^{\prime \prime} \times 50^{\prime \prime}$, is plotted in the bottom right corner. The noise level is $5.5 \mathrm{mJy}$ beam $^{-1}$.

Figure 2 a shows an average $\mathrm{HI}$ emission profile towards RCW 103 combining interferometric plus single dish data, while Fig. 2b shows an average profile of $e^{-\tau_{v}}$ over the same area, computed as described above [Equation (2)]. Strong absorption peaks appear at $-43,-17,+1$, and $+34 \mathrm{~km} \mathrm{~s}^{-1}$. All but the last feature were detected by Caswell et al. (1975) based on data obtained with the Parkes interferometer with a $2 \mathrm{~km} \mathrm{~s}^{-1}$ spectral resolution. We do not believe the absorption at $+34 \mathrm{~km} \mathrm{~s}^{-1}$ to be real because (a) absorption features should appear also from $-43 \mathrm{~km} \mathrm{~s}^{-1}$ to the tangent point at $-115 \mathrm{~km} \mathrm{~s}^{-1}$, and (b) the distance to the SNR would be approximately $20 \mathrm{kpc}$, which gives an unrealistic size and expansion velocity.

For comparison, we obtained ATCA spectra towards the strong Galactic radio sources present in the field G332.7-0.6 and G332.2-0.4 (Fig. 1), placed within $15^{\prime}$ of RCW 103, and found that absorption features appear only in the velocity ranges from 0 to -50 and 0 to $-58 \mathrm{~km} \mathrm{~s}^{-1}$ respectively.

To solve this puzzle, we followed the method employed by Dickey et al. (2003). We constructed a new Hi cube without subtracting the radio continuum and removing all baselines shorter than $1 \mathrm{k} \lambda(210 \mathrm{~m})$. In this way, all Hi structures larger than $\sim 4^{\prime}$ are filtered out. The term involving $T_{\mathrm{s}}$ in Equation (1) can thus be neglected. The data cube was further weighted by the radio continuum. We then computed $e^{-\tau_{v}}$ using Equation (1). The resulting profile is plotted in Fig. 2c.

This filtering method succeeded in removing most of the Hi emission, as is apparent when comparing Figs. 2b and $2 \mathrm{c}$. The absorption feature at $-43 \mathrm{~km} \mathrm{~s}^{-1}$ is confirmed, while the feature at $+34 \mathrm{~km} \mathrm{~s}^{-1}$ has disappeared. This implies that the latter was produced by self absorption on a scale between $4^{\prime}$ and $\sim 30^{\prime}$.

The absorption feature at $-43 \mathrm{~km} \mathrm{~s}^{-1}$ corresponds to a lower distance limit of $3.1 \mathrm{kpc}$ according to the Galactic

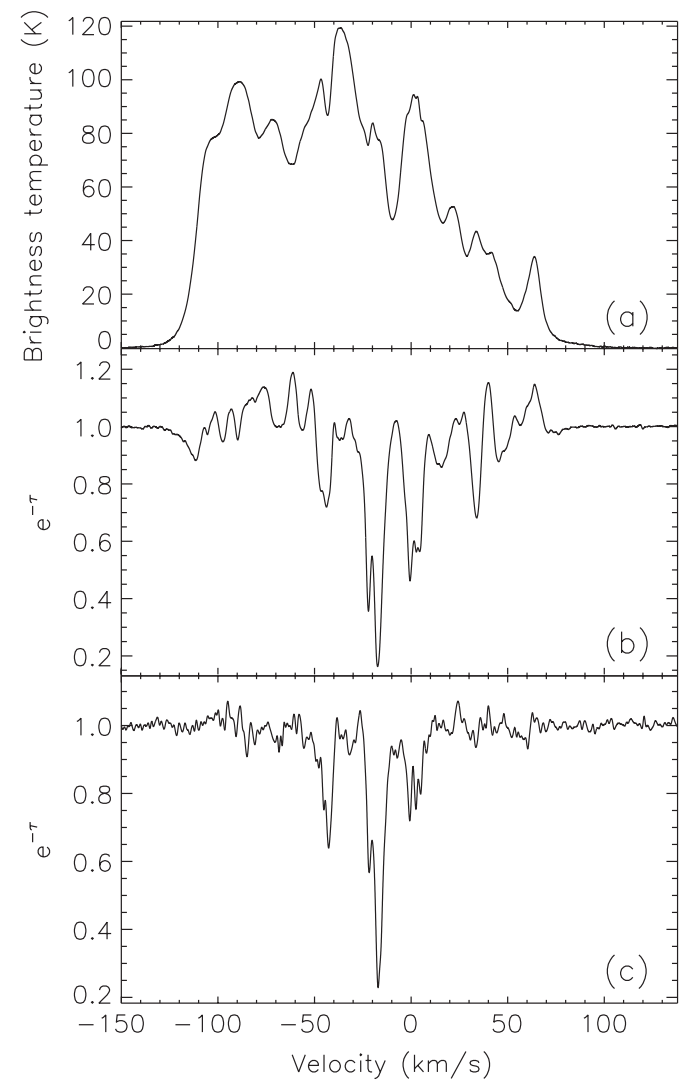

Figure 2 (a) Average HI emission profile towards RCW 103 using interferometric and single dish data. (b) Absorption profile towards RCW 103 based on interferometric ATCA data. (c) Same as (b) but excluding baselines shorter than $1 \mathrm{k} \lambda$. In all cases, the velocity resolution is $1 \mathrm{~km} \mathrm{~s}^{-1}$.

rotation model of Fich, Blitz, \& Stark (1989). We do not see any absorption against the emission peak at $\sim-75 \mathrm{~km} \mathrm{~s}^{-1}$, even though it has a brightness temperature of $\sim 80 \mathrm{~K}$. Thus the upper distance limit is $4.6 \mathrm{kpc}$. The lower distance coincides with the near side of the Scutum-Crux arm (Georgelin \& Georgelin 1976), and will be assumed throughout the paper.

\subsection{HI Associated with RCW 103}

We estimated the Hi column density towards 1E 1613485055 by integrating the foreground $\mathrm{HI}$ brightness temperature of the interferometric plus single-dish data up to $-43 \mathrm{~km} \mathrm{~s}^{-1}$. The value obtained depends on the lower limit adopted for the velocity interval in the integration since, although the local gas is supposed to lie at a systemic velocity of $0 \mathrm{~km} \mathrm{~s}^{-1}$, turbulence may cause departures of about $7 \mathrm{~km} \mathrm{~s}^{-1}$ from this value. Besides, due to the distance ambiguity, it is possible that some background gas is included in the integration, leading to an overestimation of $N_{\mathrm{HI}}$. At $v \simeq-10 \mathrm{~km} \mathrm{~s}^{-1}$, the line of sight crosses the far side of the Scutum-Crux arm. Taking into account these considerations, we integrate the brightness temperature between $\sim+3.5$ and $-43 \mathrm{~km} \mathrm{~s}^{-1}$ and estimate the Hi column density to be $N_{\mathrm{HI}} \lesssim 6 \times 10^{21} \mathrm{~cm}^{-2}$. The implications of this determination will be discussed in section 4 . 


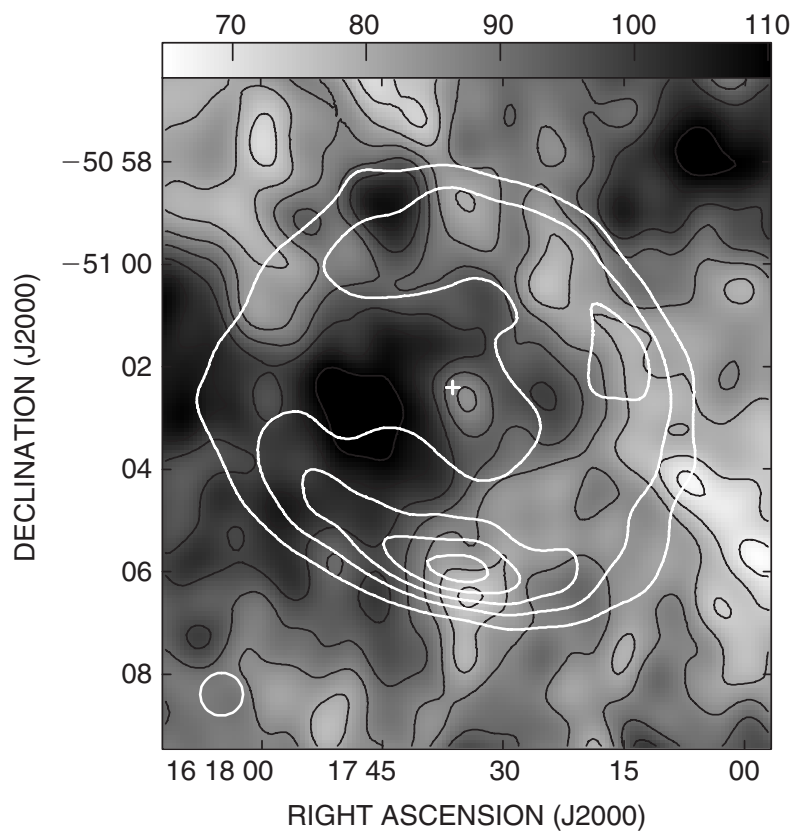

Figure 3 Gray-scale and contour image of the average Hi emission between -46.1 and $-53.5 \mathrm{~km} \mathrm{~s}^{-1}$ towards RCW 103. The rms is $2 \mathrm{~K}$. The beam, $50^{\prime \prime} \times 50^{\prime \prime}$, is plotted as a white open circle in the bottom left corner. The brightness-temperature scale is indicated on top of the image in units of $\mathrm{K}$, while the contour levels vary from $68 \mathrm{~K}$ in steps of $6 \mathrm{~K}$. For comparison, a few representative contours of the radio continuum emission are included as white lines. The cross indicates the position of 1E 161348-5055 as given by Garmire et al. (2000a).

An inspection of the Hi images at velocities around $-43 \mathrm{~km} \mathrm{~s}^{-1}$ revealed that $1 \mathrm{E} 161348-5055$ lies inside a local Hi depression which is present in all channels between -46.1 and $-53.5 \mathrm{~km} \mathrm{~s}^{-1}$. In Figure 3, an image of the average HI emission within this velocity interval is shown. The Hi depression, which attains its minimum at $\mathrm{RA}=16^{\mathrm{h}} 17^{\mathrm{m}} 34^{\mathrm{s}} .8$, Dec $=-51^{\circ} 2^{\prime} 40^{\prime \prime}(\mathrm{J} 2000)$, is elongated, with a minor-to-major axis ratio of $\sim 0.7$, and has a mean diameter of $\sim 64^{\prime \prime}$ ( $1 \mathrm{pc}$ at a distance of $3.1 \mathrm{kpc}$ ). $1 \mathrm{E} 161348-5055$ is $20^{\prime \prime}(0.3 \mathrm{pc})$ away from the centre of the hole. We note that due to the proximity to the Galactic Plane, our data show several similar HI depressions at different locations and velocities. However, the coincidence in position and velocity makes an association between the $\mathrm{CCO}$ and this Hi feature very likely.

In what follows, we analyse possible origins for this HI void. If the Hi minimum is produced because of a real absence of $\mathrm{HI}$, then the missing mass is estimated to be $0.3 \mathrm{M}_{\odot}$. If instead it is produced by hot HI gas selfabsorbed by a cooler foreground, as proposed for the CCO 1E 1207.4-5209 (Giacani et al. 2000), then we can follow the method described by Schwarz et al. (1995) to estimate an upper limit for the temperature of the hot neutral hydrogen gas.

If self-absorption is considered, then Equation (1) can be re-written as [cf. Equation (3) in Schwarz et al. 1995]

$$
T_{\mathrm{L}_{v}}=\left(T_{\mathrm{s}}-T_{\mathrm{bg}}\right) A_{v}-T_{\mathrm{c}} A_{v},
$$

with $A_{v}=\left(1-e^{-\tau_{v}}\right)$, and where $T_{\mathrm{bg}}$ is the temperature of the background hot Hi. Assuming that $A_{v}$ is uniform across the continuum source, then $T_{\mathrm{L}_{v}}$ is a linear function of $T_{\mathrm{c}}$ with slope $\left(-A_{v}\right)$ and a zero offset $\left(T_{\mathrm{s}}-T_{\mathrm{bg}}\right) A_{v}$. The HI hole attains its minimum emission at $v=-46.1 \mathrm{~km} \mathrm{~s}^{-1}$. We compared the brightness temperature of the ATCA HI data at this velocity with the continuum emission and fitted a straight line to the distribution. To avoid confusion possibly introduced by regions of low continuum emission, all values of $T_{\mathrm{c}}$ under $350 \mathrm{mJy}^{\text {beam }}{ }^{-1}$ were clipped. We determined that $A_{v} \simeq 0.195$, which implies that $\tau_{v} \simeq 0.2$. To estimate the spin temperature, it must be noted that for a single dish measurement towards a region with no continuum emission, the brightness temperature of the line is $T_{\mathrm{L}_{v}}=T_{\mathrm{S}} A_{v}$ (Schwarz et al. 1995). We averaged the Parkes data in a box around RCW 103 (excluding the contribution from the SNR) and found that $\left\langle T_{\mathrm{L}_{v}}\right\rangle \leq 100 \mathrm{~K}$, hence $T_{\mathrm{s}} \leq 20 \mathrm{~K}$. Finally, at the location of 1E 161348-5055, the interferometric data give $T_{\mathrm{c}}=44 \mathrm{~K}$ and $T_{\mathrm{L}_{v}}=-30 \mathrm{~K}$. Thus, applying Equation (3) to the emission towards the $\mathrm{CCO}$, the hot Hi gas in the hole is found to have a temperature of $\leq 130 \mathrm{~K}$. Such a temperature is unrealistically low at the interior of a SNR, therefore we discard the possibility that the HI minima observed around CCOs are produced by self-absorption.

\subsection{Radio Continuum Emission}

The rotational energy of pulsars is dissipated via a magnetised relativistic wind composed of electrons and positrons. The shock front between this wind and the ambient medium can give rise to a synchrotron emitting bubble (known as a pulsar wind nebula, PWN). The detectability of such a PWN depends on the density of the ambient medium and the pulsar parameters. The majority of pulsars do not have detectable PWN (Gaensler et al. 2000). For a very young pulsar inside a SNR, the luminosity $L$ of the PWN is a strong function of the initial period of the pulsar, $P_{0}\left(L \propto P_{0}^{-5}\right.$, Reynolds \& Chevalier 1984).

We do not detect a PWN at the position of 1E 161348 5055. Rather, the radio continuum emission in RCW 103 has a minimum near the CCO (Fig. 4). To investigate if there is a PWN around the CCO that could be beam-diluted at the resolution of 50", we constructed another continuum image using only the longest baselines. This image, with a resolution of $6.2 \times 4$.' 6 , shows no emission at the position of the CCO down to a level of $5 \sigma=1 \mathrm{mJy}^{\text {beam }}{ }^{-1}$. This limit is not very constraining - it may imply either that the spin period of 1E 161348-5055 is not particularly short or that the PWN is not well confined in the interior of the SNR.

\section{Discussion}

\subsection{Spectral Model}

Gotthelf et al. (1997) investigated different fits to the Xray spectrum of 1E 161348-5055 using a nonequilibrium ionisation plasma model combined alternatively with a blackbody, a power-law, and a thermal bremsstrahlung 
continuum component. The best fits were achieved with Hi column densities of 5, 31, and $16 \times 10^{21} \mathrm{~cm}^{-2}$ respectively, although a fixed value of $N_{\mathrm{HI}}=6.7 \times 10^{21} \mathrm{~cm}^{-2}$ also gives reasonable fits in all three cases. The value of $N_{\mathrm{HI}}$ that we estimated from the present observations, $N_{\mathrm{HI}} \leq 6 \times 10^{21} \mathrm{~cm}^{-2}$, clearly favours the blackbody solution in this case.

However, since 1997, there is growing evidence to indicate that 1E 161348-5055 might be an accreting binary system. Chandra and ASCA observations revealed a sinusoidal light curve with a period of $\sim 6.4 \mathrm{~h}$ (Garmire et al. 2000a). Even though subsequent observations failed to detect the modulation again (Garmire et al. 2000b) due to pile-up in the detectors from the very high X-ray flux, the detection of two partial dips separated by $180^{\circ}$ in phase in the light curve, and a possible near-IR counterpart about $2^{\prime \prime}$ away from the nominal Chandra position, suggest that 1E 161348-5055 may be powered by accretion likely from a low-mass companion in a binary system (Sanwal et al. 2002). Finally, Becker \& Aschenbach (2002)

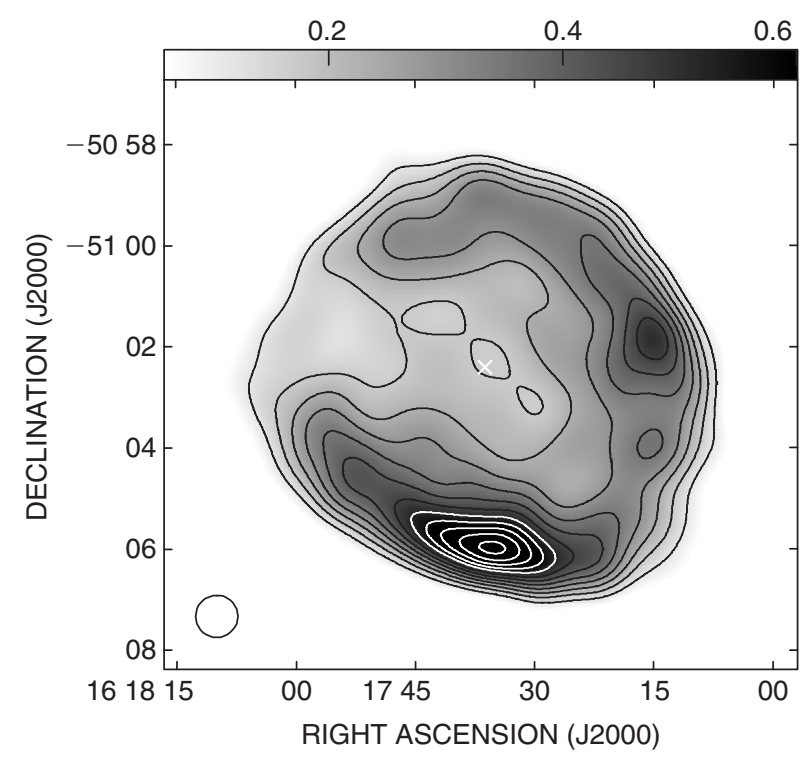

Figure 4 Gray-scale and contour image of RCW 103 at the radio continuum frequency of $1384 \mathrm{MHz}$. The gray scale is shown on top of the image in units of $\mathrm{Jy} \mathrm{beam}^{-1}$. The contours are plotted in steps of $7.5 \%$ of the peak intensity, $768 \mathrm{mJy}^{\text {beam }^{-1}}$, starting at $15 \%$. For clarity, white lines are used over regions of darker background. The cross shows the position of 1E 161348-5055 as given by Garmire et al. (2000a). The noise level is $5.5 \mathrm{mJy}$ beam $^{-1}$. discovered an eclipse $3 \mathrm{~h}$ after the start of their observations using XMM-Newton data. This would make $1 \mathrm{E}$ 161348-5055 the first accreting binary detected inside a SNR. Becker \& Aschenbach (2002) also obtained an X-ray spectrum towards $1 \mathrm{E} 161348-5055$ and found that a simple blackbody model describes the data almost up to $\sim 5 \mathrm{keV}$ but not beyond. They obtain a best fit with a double blackbody, but the required $N_{\mathrm{HI}}$ of $\leq 18 \times 10^{21} \mathrm{~cm}^{-2}$ is incompatible with our results.

We note that all the blackbody models imply temperatures as high as $8-10 \times 10^{6} \mathrm{~K}$ (Becker \& Aschenbach 2002) and a luminosity of $\sim 10^{34} \mathrm{ergs} \mathrm{s}^{-1}$ (Gotthelf et al. 1997), at least twice the values predicted by theoretical models (see Gotthelf et al. 1997 and references therein). In general, fitting NS models to the compact sources inside SNRs presents the problem that the derived parameters (temperature and luminosity) are far larger than normally expected (Lloyd, Hernquist, \& Heyl 2002). In addition, estimates of X-ray emitting areas based on blackbody models are usually too small to be the size of a NS (e.g. Becker \& Aschenbach 2002). One possible solution is proposed by Heyl \& Hernquist (1998), who show that a hot blackbody can be mimicked if the cooling NS model includes an ultramagnetised $\operatorname{star}\left(B \sim 10^{15} \mathrm{G}\right)$ with an accreted hydrogen atmosphere. This explanation was also invoked for other CCOs such as RX J0822-43 in Puppis A (Zavlin et al. 1999) and 1E 1207.4-5209 in G296.5+10.0 (Zavlin et al. 1998). Even in non-magnetic atmospheres, the effect of $\mathrm{H}$ or $\mathrm{He}$ atmospheres is to deviate the high energy spectrum in such a way that the effective temperatures as given by blackbody models could be overestimated by a factor of two (Zavlin, Pavlov, \& Shibanov 1996).

In summary, it seems most likely that 1E 161348-5055 is a binary system and that fits to the X-ray spectrum need to be re-visited. On the other hand, it is still possible that this system is a single neutron star. The constraints we have put on $N_{\mathrm{HI}}$ show that it is very hot for its age, and may indicate exotic atmospheric processes at work.

\subsection{The HI Depression}

The present study reveals the third case in which a CCO associated with a SNR is located inside an HI depression. The other two cases are 1E 1207.4-5209 in the SNR G296.5+10.0, and RX J0822-4300 in Puppis A. In Table 1 we compare the main parameters derived for

Table 1. Parameters of the HI depressions around CCOs

\begin{tabular}{|c|c|c|c|}
\hline $\begin{array}{l}\mathrm{CCO} \\
\text { (associated SNR) }\end{array}$ & $\begin{array}{c}1 \mathrm{E} 1207.4-5209^{a} \\
(\mathrm{G} 296.5+10.0)\end{array}$ & $\begin{array}{l}\text { RX J0822-4300 } \\
\text { (Puppis A) }\end{array}$ & $\begin{array}{c}1 \mathrm{E} 161348-5055^{c} \\
(\mathrm{RCW} \mathrm{103)}\end{array}$ \\
\hline Mean angular diameter $\left({ }^{\prime}\right)$ & 5.3 & 2.3 & 1.1 \\
\hline Mean linear diameter $(\mathrm{pc})$ & 3.2 & 1.5 & 1.0 \\
\hline Minor/major axis ratio & 0.8 & 0.8 & 0.7 \\
\hline CCO offset from centre $\left(^{\prime \prime}\right)$ & 30 & 37 & 20 \\
\hline CCO offset from centre (pc) & 0.3 & 0.4 & 0.3 \\
\hline Missing mass $\left(\mathrm{M}_{\odot}\right)$ & - & 0.1 & 0.3 \\
\hline
\end{tabular}


the three Hi depressions. For G296.5+10.0, only the size and velocity width are given in Giacani et al. (2000) and thus the remaining parameters, where possible, have been estimated directly from the images presented in their paper. In computing the minor to major axis ratio, the beam elongation was taken into account.

A number of similarities can be found between the three cases. All Hi cavities appear to have the same elongation, and the CCOs are off-centred by similar distances. In the two cases where the missing mass was computed, a similar value was obtained. On the other hand, the size of the Hi hole in G296.5+10.0 is larger than the other two holes by more than a factor of two. This discrepancy may be related to the beam size of the observations, which is almost four times larger than those of the Puppis A and RCW 103 data. The main difference between the three cases is given by Puppis A, which presents two lobelike HI depressions emerging from its associated $\mathrm{CCO}$ at the systemic velocity of the SNR (Reynoso et al. 2003). The present observations do not reveal any similar morphology around 1E 161348-5055.

In what follows we investigate whether the Hi depression around 1E 161348-5055 can be a swept up hole. The rate of energy production needed to set the missing mass in the cavity into motion with velocity $V=r / t$ is

$$
\dot{E}_{k}=3 \times 10^{47} \mathrm{Mr}^{2} \mathrm{t}^{-3} \mathrm{erg} \mathrm{s}^{-1},
$$

where $M$ is the evacuated mass in units of $\mathrm{M}_{\odot}, r$ is the radius of the hole in pc, and $t$ is the age of the $\mathrm{CCO}$, equal to the age of the host SNR, in years. Replacing in Equation (4) the radius and missing mass computed in section 3, and assuming that the age of RCW 103 is $2000 \mathrm{yr}$ (Carter et al. 1997), we obtain $\dot{E}_{k}=3_{-2}^{+20} \times 10^{36} \mathrm{erg} \mathrm{s}^{-1}$, where the quoted errors allow for ages of 1000 and 3000 yrs old. The spin down energy loss has not been measured for 1E 161348-5055, but if it is similar to the values observed in other CCOs (energy loss rates between $\sim 1 \times 10^{36} \mathrm{erg} \mathrm{s}^{-1}$ and $1.5 \times 10^{37} \mathrm{erg} \mathrm{s}^{-1}$; Slane et al. 1997; Brazier \& Johnston 1999), then a complete conversion of the rotational energy into kinetic energy of the surrounding medium can account for the observed Hi hole.

An alternative possibility is that the depression does not contain swept up, low density gas, but is filled with $\mathrm{HI}$ gas heated up by the CCO at temperatures higher than the surroundings. In that case, the depression should contain enhanced ionised hydrogen (i.e. it would form a small HII region), and this is not observed in the radiocontinuum image. We would, however, expect to see infra-red emission from such a region but a search in mid- and nearinfrared wavelengths using data from the Midcourse Space Experiment (MSX) and the Two Micron All Sky Survey (2MASS) yielded negative results. In conclusion, we find that the swept-up cavity provides a more convincing explanation for the observed Hi minimum.

\section{Conclusions}

In this paper, we present the third case in which a $\mathrm{CCO}$ lies at a local Hi minimum at a velocity compatible with the systemic velocity of the host SNR. We have shown that self-absorption does not provide a satisfactory explanation for this kind of feature, as was proposed for 1E 1207.4 5209 (Giacani et al. 2000). Instead, it is possible that the HI depression is a swept up hole, where $\sim 0.3 \mathrm{M}_{\odot}$ of neutral gas has been evacuated. We have found a number of similarities between the three Hi holes around CCOs detected so far, such as the elongation, the missing mass, and the off-centred position of the CCOs. We did not detect any synchrotron nebula around the $\mathrm{CCO}$ down to a level of $1 \mathrm{mJy}^{\text {beam }}{ }^{-1}$.

The present data allow us to constrain the Hi column density to be $N_{\mathrm{HI}} \sim 6 \times 10^{21} \mathrm{~cm}^{-2}$. This column density favours the blackbody model of Gotthelf et al. (1999), however the derived temperature is too high to be explained by standard NS cooling models. Instead, it appears most likely that $1 \mathrm{E} 161348-5055$ is an accreting binary, and may constitute the first case in which such a system occurs in the interior of a SNR.

\section{Acknowledgments}

We acknowledge the invaluable help of Naomi McClureGriffiths, who provided the data from the SGPS and assisted us in combining them with the ATCA observations. E.M.R acknowledges a Postdoctoral External fellowship from CONICET, Argentina. This publication makes use of data products from the 2MASS, which is a joint project of the University of Massachusetts and the Infrared Processing and Analysis Center, funded by the National Aeronautics and Space Administration and the National Science Foundation. The MOST is operated by the University of Sydney with support from the Australian Research Council and the Science Foundation for Physics within the University of Sydney. This research was partially funded through CONICET grant 4203/96 and grant UBACYT A013. The National Radio Astronomy Observatory is a facility of the National Science Foundation operated under a cooperative agreement by Associated Universities, Inc. The Australia Telescope is funded by the Commonwealth of Australia for operation as a National Facility managed by the CSIRO.

\section{References}

Becker, W., \& Aschenbach, B. 2002, in Proc. of the 270. WE-Heraeus Seminar on Neutron Stars, Pulsars and Supernova Remnants, eds. W. Becker, H. Lesch, \& J. Trümper, (Garching bei München: MPE), 64

Becker, W., Trümper, J., Hasinger, J., \& Aschenbach, B. 1993, in Isolated Pulsars, eds. K. A. van Riper, R. I. Epstein, \& C. Ho (Cambridge: Cambridge University Press), 116

Brazier, K. T. S., \& Johnston, S. 1999, MNRAS, 305, 671

Burton, M. G., \& Spyromilio, J. 1993, PASA, 10, 327

Carter, L. M., Dickel, J. R., \& Bomans, D. J. 1997, PASA, 109, 990

Caswell, J. L., Murray, J. D., Roger, R. S., Cole, D. J., \& Cook, D. J. 1975, A\&A, 45, 239

Caswell, J. L., Haynes, R. F., Milne, D. K., \& Wellington, K. 1980, MNRAS, 190, 881

Dickel, J. R., Green, A., Ye, T., \& Milne, D. K. 1996, AJ, 111, 340

Dickey, J. M., McClure-Griffiths, N. M., Gaensler, B. M., \& Green, A. J. 2003, ApJ, 585, 801 
Fich, M., Blitz, L., \& Stark, A. A. 1989, ApJ, 342, 272

Frail, D. A. 1998, in The Many Faces of Neutron Stars, eds. R. Buccheri, J. van Paradijs, \& M. A. Alpar (NATO ASI Ser. C, 515; Dordrecht: Kluwer), 179

Gaensler, B. M., Stappers, B. W., Frail, D. A., Moffett, D. A., Johnston, S., \& Chatterjee S. 2000, MNRAS, 318, 58

Garmire, G. P., Pavlov, G. G., Garmire, A. B., \& Zavlin, V. E. 2000a, IAU Circ. 7350

Garmire, G. P., Garmire, A. B., Pavlov, G. G., \& Burrows, D. N. 2000b, AAS HEAD Meeting 32, \#32.11

Georgelin, Y. M., \& Georgelin, Y. P. 1976, A\&A, 49, 57

Geppert, U., Page, D., \& Zannias, T. 1999, A\&A, 345, 847

Giacani, E. B., Dubner, G. M., Green, A. J., Goss, W. M., \& Gaensler, B. M. 2000, AJ, 119, 281

Gotthelf, E. V., Petre, R., \& Hwang, U. 1997, ApJ, 487, L175

Gotthelf, E. V., Petre, R., \& Vasisht, G. 1999, ApJ, 514, L107

Heyl, J. S., \& Hernquist, L. 1998, MNRAS, 298, 17

Leibowitz, E. M., \& Danziger, I. J. 1993, MNRAS, 204, 273

Lloyd, D. A., Hernquist, L., \& Heyl, J. S. 2002, in ASP Conf. Ser. 271, Constraining the Birth Events of Neutron Stars, eds. P. O. Slane \& B. M. Gaensler (San Francisco: ASP), 323

McClure-Griffiths, N. M., Green, A. J., Dickey, J. M., Gaensler, B. M., Haynes, R. F., \& Wieringa, M. H. 2001, ApJ, 551, 394

Oliva, E., Moorwood, A. F. M., \& Danziger, I. J. 1990, A\&A, 240,453

Oliva, E., Moorwood, A. F. M., Drapatz, S., Lutz, D., \& Strum, E. 1999, A\&A, 343, 9430

Pavlov, G. G., Zavlin, V. E., \& Trümper, J. 1999, ApJ, 511, L45
Pavlov, G. G., Sanwal, D., Garmire, G. P., \& Zavlin, V. E. 2002, in ASP Conf. Ser. 271, Neutron Stars in Supernova Remnants, eds. O. Slane \& B. M. Gaensler, 247 (astro-ph/0112322)

Reynolds, S. P., \& Chevalier, R. S. 1984, ApJ, 278, 630

Reynoso, E. M., Green, A. J., Johnston, S., Dubner, G. M., Giacani, E. B., \& Goss, W. M. 2003, MNRAS, 345, 671

Ruiz, M. T. 1983, AJ, 88, 1210

Sanwal, D., Garmire, G. P., Garmire, A., Pavlov, G. G., \& Mignani, R. 2002, BAAS, 200, 7201

Sault, R. J., Teuben, P. J., \& Wright, M. C. H. 1995, in ASP Conf. Ser. 77, Astronomical Data Analysis Software and Systems IV, eds. R. A. Shaw, H. E. Payne, \& J. J. E. Hayes (San Francisco: ASP), 433

Schwarz, U. J., Goss, W. M., Kalberla, P. M., \& Benaglia, P. 1995, A\&A, 299, 193

Slane, P., Seward, F. D., Bandiera, R., Torii, K., \& Tsunemi, H. 1997, ApJ, 485, 221

Tuohy, I. R., Córdova, F. A., Garmire, G. P., Mason, K. O., Charles, P. A., Walter, F. M., \& Clark, D. H. 1979, ApJ, 230, 37

Tuohy, I. R., \& Garmire, G. P. 1980, ApJ, 239, L107

Tuohy, I. R., Garmire, G. P., Manchester, R. N., \& Dopita, M. A. 1983, ApJ, 268, 778

van den Bergh, S., Marscher, A., \& Terzian, Y. 1973, ApJS, 26, 19

Vasisht, G., Kulkarni, S. R., Anderson, S. B., Hamilton, T. T., \& Kawai, N. 1997, ApJ, 476, L43

Zavlin, V. E., Pavlov, G. G., \& Shibanov, Yu. A. 1996, A\&A, 315, 141

Zavlin, V. E., Pavlov, G. G., \& Trümper, J. 1998, A\&A, 331, 821

Zavlin, V. E., Trümper, J., \& Pavlov, G. G. 1999, ApJ, 525, 959 